https://dx.doi.org/10.4314/ijs.v20i1.4

Ife Journal of Science vol. 20, no. 1 (2018)

\title{
A COMPARATIVE ANALYSIS OF GLUCOSE OXIDASE METHOD AND THREE POINT-OF-CARE MEASURING DEVICES FOR GLUCOSE DETERMINATION.
}

\author{
"Ekun, O. A., Ogunyemi G. A, Azenabor, A. and Akinloye O. \\ Department of Medical Laboratory Science, College of Medicine, University of Lagos, Nigeria. \\ *Corresponding Author: E-mail: ayodele1619.oe@gmail.com +2348033151619 \\ (Received: $5^{\text {th }}$ October, 2017; Accepted: $12^{\text {th }}$ November, 2017)
}

\section{ABSTRACT}

\begin{abstract}
Diabetes mellitus is gradually becoming a major health burden in Nigeria, partly due to westernization of our diet and life style. It affects both young and old people and when not properly managed may lead to complications. Thus there is need for quick, convenient and cheap means of monitoring blood glucose level for optimum management. This study aimed at comparing some point-of-care testing devices with a laboratory method. A total of 150 volunteers participated in this study. Laboratory glucose determination was carried out on plasma samples using glucose oxidase method while finger prick whole blood was used for point-of-care testing methods (Accu Chek, On Call Plus, One Touch UltraMini). Eighty eight (88) males (58.7\%) and sixty two (62) females $(41.3 \%)$ volunteers, aged $48.37 \pm 10.89$ years participated in this study. There were significant differences in the mean value of glucose using glucose oxidase method when compared with the point-of-care testing devices $(\mathrm{p}<0.05)$. Significant positive correlations existed between mean glucose values determined by using laboratory glucose oxidase method and all point-of-care testing devices $(\mathrm{p}<0.05)$. On the other hand, analysis of variance showed a significant difference $(\mathrm{p}<0.05)$ between the glucose values from laboratory method and pointof-care testing devices used for this study. The significant difference between glucose oxidase and point-of-care testing noted in this study may suggest the importance of laboratory evaluation of plasma glucose in proper evaluation and effective management of patients with diabetes mellitus. While home monitoring with point-ofcare testing should be encouraged, it should not be absolutely relied upon as this may occasionally lead to erroneous result. The need for periodic revalidation of point-of-care methods with standard laboratory method is underscored.
\end{abstract}

Keywords: Blood glucose determination methods, Hyperglycemia, Diabetes mellitus.

\section{INTRODUCTION}

Diabetes mellitus is defined as a metabolic disorder caused by different factors characterized by a chronic high level of blood glucose with combined disturbances in carbohydrate, fat, and protein metabolism resulting from defects in insulin secretion, insulin action, or both (WHO 2006). The prevalence of diabetes mellitus continues to increase with approximately $12.9 \%$ of the population in the United States diagnosed with diabetes and an even larger portion (29.5\%) estimated to be living in a pre-diabetic state. The current prevalence of diabetes mellitus in Nigeria is between 5-6\% (International Diabetic Federation, 2013) with a current African region prevalence of between 2.1-6.7\% (International Diabetic Federation, 2015). Control of blood glucose $(B G)$ in an acceptable range remains a target for diabetes patients in both the hospital and outpatient environments (Sack et al., 2002). Glycaemic control using an insulin infusion in critically/ill patients requires frequent and rapid blood glucose monitoring (Alter and Dienes, 2009).
The accuracy of blood glucose measurements plays an important role in treatment decisions when aiming for adequate or optimum glycaemic control. In recent years, due to the technological and scientific advancement many point-of-care testing devices have been developed. These provide a means of generating rapid glucose results for emergency situations and also for home glucose monitoring in outpatient diabetic individuals. This has greatly assisted in better management of patients as the device does not require venipuncture. Also the results are obtained within seconds; however a major drawback with this device is the problem of accuracy.

Previous study of Puntmann et al., (2003) that compared the glucose results generated by pointof-care testing with the standard laboratory methods yielded divergent conclusion thus raising some doubts regarding the usefulness of point-ofcare testing. While the study of Puntman et al.,(2003) was conducted in western world, where the climatic conditions is completely different from that of Africa particularly Nigeria, it remains 
to be seen if this variation in climatic condition may impact negatively on the optimal functionality of the point-of-care testing in Nigeria. Many medical laboratories in Nigeria estimate plasma glucose using glucose oxidase method. This method for the determination of plasma glucose is considered as one of the conventional methods for blood glucose analysis; it is imperative that the point-of-care testing (POCT) devices available compare favourably with this common conventional medical laboratory methods.

However, a study by Giordano et al.,(1986) compared seven (7) commercially available pointof-care glucose monitoring devices accuracy with a reference method and observed that only three (3) out of seven (7) devices had measurement accuracy. Also, Chen et al., (2003) in their study evaluated four (4) point-of-care glucose monitoring devices blindly and observed that only two (2) devices performed with acceptable accuracy limits according to International Standard Organization standard, however, none of them achieved the 1996 American Diabetic Association (ADA) recommendations of pointof-care glucose monitoring device accuracy. All the four (4) devices showed less reliability with lower glucose values compared to normal or higher values. In another study conducted by Cohen et al., (2003), evaluation of five (5) point-ofcare glucose monitoring devices using the Clark error grid observed that four (4) out of five (5) devices met criteria for accurate clinical decision making. However, only one (1) out of five (5) devices met ADA (1996) accuracy standards.

Furthermore, study by Khan et al., (2006) showed that out of seven (7) devices from four (4) different manufacturers that were compared with reference method, only one (1) met ADA (1996) performance requirements, particularly with concern over significant disagreement with reference values within the critical hypoglycaemic range that could result in adverse clinical decision. On the other hand, study by Thomas et al., (2008) involving five (5) devices find four (4) deemed accurate enough to be used in a clinical setting based on a Clark error grid, but only two (2) provided measurement with less than $20 \%$ variation from the reference method, and only one (1) device having less than 10\% error. In addition to this, study by Rosenthal et al., (2006) in neonates for hypoglycaemia screening did not have required accuracy. There is however paucity of similar study on point-of-care glucose monitoring devices among Nigerian population.

Laboratory estimation of plasma glucose has been considered as the optimum method for measuring blood glucose. Although, there is no universally agreed reference methodology for blood glucose measurement, the hexokinase, glucose oxidase and glucose dehydrogenase enzymatic procedures are the methods in common use (Carway and Watt., 1986). The major drawbacks of laboratorybased estimation are the need for a larger volume of blood and delay in obtaining results for timely appropriate treatment (Adamkin et al., 2011).

In many developing countries like Nigeria, delay in laboratory evaluation of specimens due to a long time lag between initial sample collection and conventional test completion have caused high risk population to typically seek treatment in clinics without investigations, since most of these patients may not likely return a second time for the test, the use of point-of-care tests, may help in generating results rapidly. While this might have helped in some emergency situation, the issues concerning its accuracy may largely be a major problem. This may lead to increasing concern about the efficiency of the point of care blood glucose testing. Thus this study attempts to compare the results obtained from glucose pointof-care devices (Accu Chek, One touch Ultra Mini and On Call Plus glucometers) with that obtained from laboratory glucose oxidase method.

\section{MATERIALS AND METHODS}

\section{Volunteer selection}

A total of 150 confirmed diabetic patients from diabetic clinic participated in the study. Participants were patients who required glucose monitoring for therapeutic care. They were all referred to the phlebotomy unit of the Lagos University Teaching Hospital (LUTH), Idi-Araba, Lagos.

\section{Ethical consideration}

Approval was obtained from the Research and Ethics Committee (ADM/DCST/HREC/2175) 
of the Lagos University Teaching Hospital (LUTH) prior to the commencement of the study. Informed consent was sought and obtained from each of the participants. Only consenting volunteers were recruited for this study.

\section{Data analysis}

The data collected from the study was analyzed using SSPS version 21.0. Descriptive statistics of mean, standard deviation, percentages were determined. Association between results obtained from the various methods were evaluated using Pearson's correlation coefficient while one way analysis of variance was used to determine variation between the methods. A value of $p$ $<0.05$ was considered statistically significant for this study.

\section{Methodology \\ Glucometers}

- The test strips was inserted into the meter.

- Blood sample was obtained by pricking the patients fingertip with a lancet after sterilizing the sample area with $70 \%$ alcohol

- The drop of whole capillary blood was then placed on the reagent bonded to the paper strip.

- After a fixed time, the result appeared on a digital display screen. These meters use reflectance photometry or electrochemistry to measure the rate of the reaction or the final concentration of the products.

\section{Glucose Oxidase method.}

This is based on the principle of enzymatic oxidation of glucose in the presence of glucose oxidase. The hydrogen peroxide formed reacts under catalysis of peroxidase, with phenol and 4aminophenazone to form a red-violet quinoneimine dye as indicator (Trinder., 1963).

- 2 milliliters of venous blood was collected into fluoride oxalate bottle. It was mixed by inverting gently.

- The blood was centrifuged at the speed of 3,000 revolution per minutes (rpm) for five minutes to separate the red blood cell from the plasma.

- $\quad 1000 \mu \mathrm{l}$ of glucose oxidase reagent was pipetted into each clean test tube labelled 'standard' and 'test' was placed in a rack.

- $10 \mu \mathrm{l}$ of standard and plasma were added to their respective test tubes and mixed properly.

- The mixture was incubated at room temperature for 10-15 $\mathrm{min}$.

- It was read at $500 \mathrm{~nm}$ using a colorimeter.

\section{RESULTS AND DISCUSSION.}

A total of one hundred and fifty (150) participated in the study (Table 1), which included 88 males $(58.7 \%)$ and 62 females $(41.3 \%)$. Their age range varied from 22-68 years, with a mean age of $48.37 \pm 10.89$ years. The age range of the volunteered participants in this study underscores the fact that diabetes mellitus is common among adult Nigerians. It has been documented by the International Diabetes Federation (IDF), (2013 and 2015), that in African region, diabetes mellitus affects both children and adults with the adult age seen to be between 20-79 years. IDF (2015) has also reported that the prevalence by age and sex appeared more between ages 40-79 years with peak age of 50-59 years. The males were found to have higher prevalence of diabetes compared to females within the age group of 40-79 years whereas, between ages 20-39 years, the prevalence of diabetes among female appears higher than that of the males, (International Diabetes Federation, 2015).

Table 1: Socio-demographic Characteristics of the Diabetic Patients $(N=150)$

\begin{tabular}{lcccc}
\hline & N & Mean \pm SD & Percentage $\%$ & Cummulative $\%$ \\
\hline Age (years) & & & & $100 \%$ \\
Male & 88 & $48.371 \pm 10.89$ & $100 \%$ & 58.7 \\
Female & 62 & & $58.7 \%$ & 41.3 \\
\hline
\end{tabular}


Table 2: Comparative analysis of glucose determination using glucose oxidase method and three different types of glucometers.

\begin{tabular}{lccrc}
\hline Method & Number of participants' mean \pm S.D $(\mathrm{mmol} / \mathrm{L})$ & t value & $\mathrm{p}$ Value \\
\hline Glucose oxidase & 150 & $5.40 \pm 1.46$ & & \\
Accu Chek & 150 & $5.24 \pm 1.39$ & 3.659 & $0.0001^{*}$ \\
One Touch UltraMini & 150 & $6.11 \pm 1.56$ & 11.702 & $0.0001^{*}$ \\
On Call Plus & 150 & $6.16 \pm 1.48$ & 12.398 & $0.0001^{*}$ \\
\hline
\end{tabular}

Significant level: $\mathrm{p}<0.05$

The glucose values (Table 2) obtained from Accu chek $(5.24 \pm 1.39 \mathrm{mmol} / \mathrm{L})$ was significantly lower $(p=0.0001)$ than those from laboratory glucose oxidase method $(5.40 \pm 1.46 \mathrm{mmol} / \mathrm{L})$. On the other hand, values from One Touch UltraMini $(6.11 \pm 1.56 \mathrm{mmol} / \mathrm{L})$ and $\mathrm{On}$ Call Plus glucometers $(6.16 \pm 1.48 \mathrm{mmol} / \mathrm{L})$ were observed to be significantly higher $(\mathrm{p}=0.0001)$ than those from the laboratory glucose oxidase method $(5.40 \pm 1.46 \mathrm{mmol} / \mathrm{L})$. We observed a significant decrease in the mean value of glucose determined using Accu Chek glucometer when compared to laboratory glucose oxidase method $(p<0.05)$. However with other point-of-care testing (POCT) devices a significant increase $(p<0.05)$ was observed when compared to the laboratory glucose oxidase method. Our observation with these three point-of-care glucometers agreed with the previous study of Cook., (2001) who determined the level of agreement between glucose values obtained by laboratory analysis and with a point-of-care device for blood from two different sources- finger stick and a central venous catheter. Also this study is in consonant with the study of Van-den Berghe et al., (2001) where a significant difference was observed between glucose value obtained from POCT and standard laboratory glucose method. On the contrary, this study was observed to be at variance with the studies of Umpierrez et al., (2002) and Lacara et al., (2007) where a non-significant difference was observed between the glucose values of POCT and laboratory. The possible explanation for this observation could be due to variation in the degree of sensitivity of the different types of POCTs used in these various studies.

Glucose concentration may be determined in whole blood, plasma, or serum samples, the glucose point-of-care devices use whole capillary blood for glucose estimation, while plasma or serum is required for standard laboratory methods for glucose estimation. Studies have shown that plasma or serum glucose is higher than glucose present in whole blood (Sacks, 2008). This is because plasma or serum has higher water content than whole blood, so there is more dissolved glucose in plasma or serum compared with whole blood, and readings are $15-20 \%$ higher. Point-ofcare blood glucose devices measure whole blood which is a complex medium of plasma and red blood cells. Although glucose is distributed within both fractions, point-of-care devices essentially interrogate plasma and thus are generally calibrated to yield a plasma glucose equivalent (Saudek et al., 2006). It was observed that of all the three point-of-care testing devices used in this study, only Accu Chek glucometer had a mean glucose value that was slightly lower than the laboratory glucose oxidase method by $2.96 \%$. Another contributing factor is that whole blood consists of both red cells and leucocytes, which contains glycolytic enzymes, leading to the consumption and reduction of glucose in a sample of whole blood (McMillin, 1990). However, it should be noted that this study was carried out within few minutes of sampling through finger prick, hence glycolytic enzyme effect does not apply.

American Diabetes Association (ADA), (1987) consensus statement recommended that the acceptable error for point-of-care glucose monitoring devices from all sources (user, analytical) should be less than $10 \%$ for glucoses ranging from 30 to $400 \mathrm{mg} / \mathrm{dl} \quad(1.67-22.22$ $\mathrm{mmol} / \mathrm{l}$ ) at all times. This ADA consensus statement also recommended that glucose measurements should not differ by more than $15 \%$ from values obtained by a laboratory 
reference method. In this study we observed that Accu Chek differ from glucose oxidase by $2.96 \%$ while One Touch UltraMini and On Call Plus differ from glucose oxidase by $13.15 \%$ and $14.07 \%$ respectively, suggesting that Accu Chek gave the closest result to the laboratory glucose oxidase method. From this observation, all the point-of-care testing devices evaluated in this study were observed to be within the acceptable measuring limit of variation based on ADA, (1987) consensus.

Factors that might have affected the performance of the glucose point-of-care devices used are patient's haematocrit level (Barreau and Buttery,
1988, Ginsberg, 2009), strip factors (variation in strips) (Teodorczyk et. al., 2012), physical factors (temperature and altitude), (Fink et. al., 2002) and intrinsic factors (e.g., sample properties, interferences and pharmacological factors) (Heinemann, 2010). Most glucometers are affected by the factors listed. In contrast, glucose estimation in hospitals and large clinics are usually performed with advanced instrument using precise and accurate methods which are well calibrated and controlled based on international standard and as such are least affected by many of the factors that commonly degrade the accuracy and precision of glucometers (Tonyushkina et. al., 2009).

Table 3: Pearsons' correlation statistical analysis of level of association between laboratory glucose oxidase method and point-of-care devices

\begin{tabular}{lccc}
\hline Association between methods & Number of participants & correlation coefficient $(\mathrm{r})$ & p value \\
\hline Glucose oxidase vs Accu check & 150 & 0.941 & $<0.0001^{*}$ \\
Glucose Oxidase vs One Touch & 150 & 0.880 & $<0.0001 *$ \\
Glucose Oxidase vs On Call Plus & 150 & 0.871 & $<0.0001^{*}$ \\
\hline
\end{tabular}

Level of significance: $\mathrm{p}<0.05$

Furthermore, table 3 describes the degree of association between the glucose values generated using different analytical methods. It was observed that Accu Chek had the strongest and significant association $(r=0.941 \mathrm{p}<0.05)$ with the laboratory glucose oxidase method. However, both One Touch UltraMini and On Call Plus glucometers all presented strong positive and significant correlation $(\mathrm{r}=0.880, \mathrm{p}<0.05, \mathrm{r}=$ $0.870, \mathrm{p}<0.05$ respectively). An evaluation of the degree of association between all the point-ofcare testing methods and laboratory glucose oxidase method using a Pearson correlation coefficient shows how closely related the results obtained were. It was observed that Accu-Chek had the strongest positive correlation with the laboratory glucose oxidase method suggesting that Accu-Chek glucometer generates results that are close to the results generated from the laboratory enzymatic glucose oxidase method. However other point-of-care devices used in this study also gave a strong and positive correlation with the laboratory glucose oxidase method. A strong, positive and significant $(\mathrm{P}<0.05)$ correlations as observed between glucose oxidase and the various point-of-care methods adopted for this study possibly suggests that all point-ofcare testing studied associate with the laboratory glucose oxidase method to a certain significant extent. 
Table 4: Measure of differences or analysis of variance between the different methods used for glucose estimation.

\begin{tabular}{lrrccc}
\hline Variables & sum of squares & df & mean square & F value & p Value \\
\hline Between Groups & 93.709 & 3 & 31.236 & 14.282 & $0.0001^{*}$ \\
Within Groups & 1303.497 & 596 & 2.187 & & \\
Total & 1397.206 & 599 & & & \\
\hline
\end{tabular}

Level of significance: $\mathrm{p}<0.05$

One way analysis of variance between the methods and devices used showed a significant variation $(\mathrm{p}=0.0001)$ between the various methods of glucose analysis (Table 4). Thus this table suggests that there exist some variations in the glucose values generated from laboratory glucose oxidase methods and the point-of-care methods. This therefore underscores the need for laboratory estimation or determination of glucose when critical decisions are to be taken in respect of accurate diagnosis and or management.

\section{CONCLUSION}

Although POCT glucose methods are often used to guide management decisions in critical care, significant differences were found between glucose values obtained with POCT devices and by laboratory glucose oxidase method, despite the strong correlation between the results. This implies that glucometers may only be used to monitor the trend of glucose level in diabetic patients, but may lead to wrong decision when actual glucose values are needed for accurate diagnosis and or management.

Acknowledgment: Authors are grateful to the staff in the phlebotomy section of Lagos University Teaching Hospital for their assistance.

Conflict of Interest: None declared.

\section{REFERENCES}

Adamkin, D. H., 2011. Committee on Fetus and Newborn. Post-natal glucose hemostasis in late preterm and term infants. Pediatrics. 127: 575-79.

Alter, D., Deines, G., 2009. Tight glycaemic control and point-of-care testing. Clin Lab Med.29(3):511-22.

American Diabetes Association, 1996. Selfmonitoring of blood glucose: clinical practice recommendations. Diabetes Care.19:S62-6.

American Diabetes Association, 1987. Consensus statement on self-monitoring of blood glucose. Diabetes Care. 10(1):93-9.

Barreau, P. B., Buttery, J. E., 1988. Effect of hematocrit concentration on blood glucose value determined on Glucometer II. Diabetes Care. 11(2): 116-118.

Carway, W. T., Watts, N. B.,. 1986. Carbohydrates in textbook of clinical chemistry chapter 6 pg775-828. Edited by Tietz, N.W., W.B. Saunders Company.

Chen, E. T., Nichols, J. H., Duh, S. H., Hortin, G., 2003. Performance evaluation of blood glucose monitoring devices. Diabetes TechnolTher. 5(5):749-68.

Cohen, M., Boyle, E., Delaney, C., Shaw, J. A., 2006. Comparison of blood glucose meters in Australia. Diabetes Res Clin Pract. 71(2):113-8.

Cook, A., Laughlin, D., Moore, M., North, D., Wilkins, K., Wong, G., Wallace-Scroggs, A., Halvorsen, L., 2009. Differences in glucose values obtained from point-ofcare glucose meters and laboratory analysis in critically ill patients. Am J Crit Care. 18(1):65-71; quiz 72. doi: 10.4037/ajcc2009626.

Fink, K. S., Christensen, D. B., Ellsworth, A., 2002. Effect of high altitude on blood glucose meter performance. Diabetes Technol Ther. 4(5):627-635.

Ginsberg, B. H., 2009. Factors affecting blood glucose monitoring: sources of errors in measurement. J Diabetes Sci Technol. 3(4):903-913.

Giordano, B. P., Hodges, C., Trash, W., Dube, W. P., Hodges, C., Swain, A., Banion, C.R., Klingensmith, G. J., 1989. Performance of 
seven blood glucose testing systems at high altitude. Diabetes Educ.15 (5):444-8.

Heinemann, L., 2010. Quality of glucose measurement with blood glucose meters at the point-of-care: relevance of interfering factors. Diabetes Technol Ther. 12(11):847-857.

International Diabetic Federation, 2015. Chapter 4; Diabetes by Region. Pages 67-97. $7^{\text {th }}$ edition.

International Diabetic Federation, 2013. Chapter 3; Regional overviews of prevalence of diabetes mellitus. pg 54-70. Sixth edition. ISBN: 2-930229-85-3.

Khan, A. I., Vasquez, Y., Gray, J., Wians, F. H., Jr., Kroll, M. H., 2006. The variability of the results between point-of-care testing glucose meters and the central laboratory analyzer. Arch Pathol Lab Med.130 (10):1527-32.

Lacara, T., Domagtoy, C., Lickliter, D., Quattrocchi, K., Snipes, L., Kuszaj, J., Prasnikar, M., 2007. Comparison of point-of-care and laboratory glucose analysis in critically ill patients. Am J Crit Care. 16(4):336-46.

McMillin, J. M., 1990. Blood Glucose. In: Walker, H. K., Hall, W. D., Hurst, J. W., editors. Clinical Methods: The History, Physical, and Laboratory Examinations. 3rd edition. Boston: Butterworths. Chapter141.Availablefrom:https://www. ncbi.nlm.nih.gov/books/NBK248/

Puntmann, I., Wosniok, W., Haeckel, R., 2003. Comparison of several point-of-care testing (POCT) glucometers with an established laboratory procedure for the diagnosis of type 2 diabetes using the discordance rate. Clin Chem Lab Med. 41(6): 809-820.

Rosenthal, M., Ugele, B., Lipowsky, G., Küster, H., 2006. The Accu-trend sensor glucose analyzer may not be adequate in bedside testing for neonatal hypoglycemia. Eur J Pediatr. 165(2):99-107.

Sacks, D. B., Bruns, D. E., Goldstein, D. E., MacLaren, N. K., McDonald, J. M., Parrott, M., 2002. Guidelines and recommendations for laboratory analysis in the diagnosis and management of diabetes mellitus. Clin Chem. 48(3):436-72.

Saudek, C. D., Derr, R. L., Kalyani, R. R., 2006. Assessing glycaemia in diabetes using selfmonitoring blood glucose and he moglobin A $1 \mathrm{c}$. JAMA. 295(14):1688-1697.

Teodorczyk, M., Cardosi, M., Setford, S., 2012. Hematocrit Compensation in Electrochemical Blood Glucose Monitoring Systems. J Diabetes Sci Technol. 6(3): $648-655$. d o i : $10.1177 / 193229681200600320$.

Thomas, L. E., Kane, M. P., Bakst, G., Busch, R. S., Hamilton, R. A., Abelseth, J. M., 2008. A glucose meter accuracy and precision comparison: the Free Style Flash versus the Accu-Chek Advantage, Accu-Chek Compact Plus, Ascensia Contour, and the BD Logic. Diabetes Technol Ther. 10(2):102-10.

Tonyushkina, K., Nichols, J. H., 2009. Glucose meters: a review of technical challenges to obtaining accurate results. $J$ Diabetes Sci Technol.3(4):971-80.

Trinder, P., 1966. Determination of glucose in blood using glucose oxidase with an alternative oxygen acceptor. Ann Clin Biochem. 6:24.

Ugege, O., Ibitoye, P. K., Jiya, N. M., 2013. Childhood diabetes mellitus in Sokoto, North-Western Nigeria: A ten year review. SahelMedJ.16:97-101

Umpierrez, G. E., Isaacs, S. D., Bazargan, N., You, X., Thaler, L. M., Kitabchi, A. E., 2002. Hyperglycemia: an independent marker of in hospital mortality in patients with undiagnosed diabetes. J Clin Endocrinol Metab. 87:978-982.

van den Berghe, G. I., Wouters, P., Weekers, F., Verwaest, C., Bruyninckx, F., Schetz, M., Vlasselaers D, Ferdinande, P., Lauwers, P., Bouillon, R., 2001. Intensive insulin therapy in critically ill patients. $N$ Engl $J$ Med. 345(19):1359-67.

World Health Organization. 2006. Definition and diagnosis of diabetes mellitus and intermediate hyperglycemia. Report of a WHO/IDF consultation. 